\title{
Volumetric modulated arc therapy planning method for supine craniospinal irradiation
}

\author{
Jianzhou Chen - Chuangzhen Chen - Todd F. Atwood • \\ Iris C. Gibbs • Scott G. Soltys • Carolina Fasola • \\ Lei Xing
}

Received: 7 March 2012 / Accepted: 23 April 2012 /Published online: 10 May 2012

(C) Springer-Verlag 2012

\begin{abstract}
Background The purpose of this study is to propose a volumetric modulated arc therapy (VMAT) treatment planning technique for supine craniospinal irradiation (CSI) in order to improve dose conformity and homogeneity, as well as the reliability of the matching of abutting fields.

Methods and materials CT datasets of a 9-year-old child and a 24-year-old adult treated with supine CSI were identified. The planning target volume (PTV) was contoured to include the whole contents of the brain and spinal canal with a uniform margin of $5 \mathrm{~mm}$. RapidArc plans were generated with two partial arcs covering the brain and the superior portion of the spinal cord and a single partial arc covering the remaining inferior portion of the spinal cord. Conformity and heterogeneity indexes ( $\mathrm{CI}$ and $\mathrm{HI}$ ) and organs at risk (OAR) dose-volume histograms were evaluated. The effect of treatment inaccuracy was simulated with intentional patient shifts of $\pm 3 \mathrm{~mm}$.

Results The CI for the pediatric and adult cases was 1.0 and 0.99 , respectively, and the HI for the two cases was 12.7 and $11.8 \%$, respectively. The mean dose to the PTV was 102.5 and $102.1 \%$, respectively, while the mean doses to the OARs were all low. The simulated \pm 3 -mm shift in patient
\end{abstract}

J. Chen · C. Chen · T. F. Atwood - I. C. Gibbs · S. G. Soltys •

C. Fasola $\cdot$ L. Xing $(\bowtie)$

Department of Radiation Oncology, Stanford University, 875 Blake Wilbur Dr,

Stanford, CA 94305-5847, USA

e-mail: lei@stanford.edu

J. Chen $(\square) \cdot$ C. Chen

Department of Radiation Oncology,

Cancer Hospital of Shantou University Medical College,

7 Raoping Road,

Shantou, Guangdong, China 515031

e-mail: cjz8080@163.com position generated an error of less than $\pm 10 \%$ of the calculated dose in the spinal cord.

Conclusions VMAT CSI was able to achieve highly conformal and homogeneous dose distributions, with minor doses to the surrounding normal tissues. Setup errors of $\pm 3 \mathrm{~mm}$ were shown to have little effect on the intended dose distribution.

Keywords Craniospinal irradiation · Volumetric modulated arc therapy $\cdot$ RapidArc $\cdot$ IMRT

\section{Introduction}

Craniospinal irradiation (CSI) is an essential component for the treatment of primary intracranial tumors with a risk of leptomeningeal spread. However, CSI is technically challenging due to the large and irregular target volume and the radiosensitivity of the spinal cord and other critical structures. Conventional three-dimensional conformal radiotherapy (3DCRT) CSI techniques require matching multiple fields, with different isocenters, to cover the intended target volume. Unfortunately, 3DCRT methods often result in dose inhomogeneity and inaccuracy at the field junction areas. A common solution to minimize the dose inhomogeneity is to use weekly interfractional manual shifts of the field junctions $[1,2]$, but there are several disadvantages to this method. First, the high-dose gradients between the matched fields have the potential to create unintentional high and/or low doses in the spinal cord when even a small setup error is introduced [3]. Second, 3DCRT CSI patients are commonly set up in the prone position to provide direct visualization of the multiple field junctions, but this position is often uncomfortable and can lead to increased patient motion during treatment. Furthermore, the oral cavity and airway are 
restricted while in the prone position, and the flexed head position is not conducive to immobilization for children requiring anesthesia. Third, interfractional manual shifts are complicated, which can exacerbate patient setup errors and lengthen the overall treatment time.

RapidArc (RA, Varian Medical Systems, CA, USA) is a type of volumetric modulated arc therapy (VMAT) that provides intensity-modulated radiation therapy (IMRT) with multi-leaf collimator (MLC), dose rate, and gantry speed modulation [4]. RapidArc has been compared with IMRT for a number of sites and malignancies commonly treated by IMRT, including brain, head and neck, prostate, anal canal, and cervical uterine, and its advantages in target coverage, treatment-time efficiency, and organs at risk (OAR) sparing have been demonstrated [5-10]. CSI, on the other hand, represents a challenging site where treatment is still predominantly done with 3DCRT because of a lack of a systematic procedure for dealing with the disease using VMAT. The objective of this study was to develop a practical VMAT technique for supine CSI and to demonstrate its advantages in improving the planning target volume (PTV) coverage and OAR sparing, as well as in reducing the sensitivity of CSI abutting fields against potential uncertainties of beam setup and intrafraction patient motion.

\section{Methods and materials}

CT datasets of a 9-year-old child (patient A) and a 24-yearold adult (patient $\mathrm{B}$ ) treated with supine 3DCRT CSI were selected. For both patients, the CT datasets were acquired using a 16-slice CT scanner (GE LightSpeed RT 16; General Electric Medical Systems, Milwaukee, WI) with the patients in a supine position. The head and shoulders of the patients were immobilized in a thermoplastic shell with the arms resting comfortably at the sides of the body. CT images were transferred to an Eclipse treatment planning system (version 8.9, Varian Medical Systems, Palo Alto, CA) for target volume and OAR contouring and subsequent treatment planning.

\section{Target volume and objectives}

The clinical target volume (CTV) was contoured to include the entire leptomeningeal region and the cranial contents (brain and meninges) and the spinal canal as described in previous studies $[11,16]$. The PTV was created by adding a uniform 5-mm volumetric margin to the CTV. OAR contours were created for the lenses, thyroid, esophagus, lungs, heart, liver, spleen, and kidneys.

The prescription doses for patients A and B were 23.4 and 30.6 Gy (delivered in 1.8-Gy fractions), respectively. Target coverage was assessed as the PTV volume receiving at least $95 \%$ of the prescribed dose $\left(V_{95} \%\right)$ and the volume receiving more than $107 \%$ of the prescribed dose $\left(V_{107} \%\right)$. The maximal dose to lenses was required to be $<8 \mathrm{~Gy}$. Considering the low prescription dose and the large target volume involved, all other OAR doses were minimized to the maximum extent.

\section{VMAT planning}

Patients were treated with $6 \mathrm{MV}$ photon beams from a Varian linac equipped with an MLC with a total of 120 leaves $(5 \mathrm{~mm}$ leaf widths at isocenter for the central $20 \mathrm{~cm}$ and of $10 \mathrm{~mm}$ leaf widths for the outer $20 \mathrm{~cm}$ ). Table 1 details the patients' characteristics, positioning, dose prescription, and arc information.

All VMAT plans were created with two isocenters. Patient $\mathrm{A}$ had isocenters placed at the levels of $\mathrm{C} 1$ and $\mathrm{T} 11$, with $25 \mathrm{~cm}$ of separation, and patient $\mathrm{B}$ had isocenters placed at the levels of $\mathrm{C} 3$ and $\mathrm{T} 12$, with $31 \mathrm{~cm}$ of separation. The full arc parameters are listed in Table 1. Two coplanar partial arcs, with opposite directions of rotation (clockwise, $\mathrm{CW}$, and counterclockwise, $\mathrm{CCW}$ ), were used to cover the superior portion of the PTV (brain and upper portion of the spinal cord). A single partial arc was used to cover the inferior portion of the PTV (majority of the spinal cord). Avoidance sectors were utilized to prevent the beams from entering the PTV through the eyes, optic nerves, arms, or lungs, and the collimator angles were alternated $\left(5^{\circ}\right.$ for

Table 1 Description of the patients, arc arrangements, and dose prescription

\begin{tabular}{|c|c|c|}
\hline Parameter & Patient A & Patient B \\
\hline Age (years)/sex & 9/male & 24/female \\
\hline Patient fixation & $\begin{array}{l}\text { Head mask, feet } \\
\text { support }\end{array}$ & $\begin{array}{l}\text { Head mask, feet } \\
\text { support }\end{array}$ \\
\hline $\begin{array}{l}\text { Length of target } \\
\text { (Sup/Inf) }\end{array}$ & $60 \mathrm{~cm}$ & $71 \mathrm{~cm}$ \\
\hline $\begin{array}{l}\text { CTV to PTV } \\
\text { margins }\end{array}$ & $5 \mathrm{~mm}$ & $5 \mathrm{~mm}$ \\
\hline $\begin{array}{l}\text { Location of } \\
\text { isocenters }\end{array}$ & a: C1/b: T11 & a: C3/b: T12 \\
\hline $\begin{array}{l}\text { Distance between } \\
\text { isocenters }\end{array}$ & $25 \mathrm{~cm}$ & $31 \mathrm{~cm}$ \\
\hline Rotation of gantry & $\begin{array}{l}\text { a: } \mathrm{CW}+\mathrm{CCW} / \mathrm{b}: \\
\mathrm{CW}\end{array}$ & $\begin{array}{l}\text { a: } \mathrm{CW}+\mathrm{CCW} / \mathrm{b}: \\
\mathrm{CW}\end{array}$ \\
\hline Angle of arc & $\begin{array}{l}179.9-180.1^{\circ} \text { except } \\
50-310^{\circ}\end{array}$ & $\begin{array}{l}179.9-180.1^{\circ} \text { except } \\
50-310^{\circ}\end{array}$ \\
\hline Angle of collimator & $\mathrm{CCW}, 5^{\circ} / \mathrm{CW}, 355^{\circ}$ & $\mathrm{CCW}, 5^{\circ} / \mathrm{CW}, 355^{\circ}$ \\
\hline$X 1 / X 2$ & $\begin{array}{c}\mathrm{a},-10.0 / 10.0 \mathrm{~b} \\
-8.0 / 8.0\end{array}$ & $\begin{array}{c}\mathrm{a},-10.0 / 10.0 \mathrm{~b} \\
-8.0 / 8.0\end{array}$ \\
\hline$Y 1 / Y 2$ & $\begin{array}{c}\mathrm{a},-16.0 / 18.0 \mathrm{~b} \\
-20.0 / 16.0\end{array}$ & $\begin{array}{c}\mathrm{a},-20.0 / 20.0 \mathrm{~b} \\
-20.0 / 20.0\end{array}$ \\
\hline Length of overlap & $8 \mathrm{~cm}$ & $10 \mathrm{~cm}$ \\
\hline MUs & 576 & 504 \\
\hline
\end{tabular}


$\mathrm{CCW}$ and $355^{\circ}$ for $\mathrm{CW}$ ) to minimize the tongue and groove effect of the MLCs. The upper and lower arcs overlapped by 8 and $10 \mathrm{~cm}$ in the pediatric and adult patients, respectively. A sample CSI PTV and arc arrangement is illustrated in Fig. 1.

A ring control structure was created $(2 \mathrm{~cm}$ volumetric expansion beginning $10 \mathrm{~mm}$ from the PTV) to increase dose conformality and control the dose gradient outside of the PTV. The upper and lower arcs were optimized together at the same time, and the plans were optimized by applying dose-volume constraints to the PTV, OAR, and control structure. In addition, the normal tissue objective tool in Eclipse was utilized to further reduce the dose outside the target. Plan optimization was performed with the progressive resolution optimizer algorithms implemented in Eclipse (version 8.9.05), and dose calculation was performed with the anisotropic analytical algorithm using a grid size of $2.5 \mathrm{~mm}$ (version 8.6.02).

\section{Evaluation of plans}

Plans were normalized so that $95 \%$ of PTV was covered by $95 \%$ of the prescription dose. Quantitative evaluation of the plans was performed in terms of dose-volume histogram (DVH) analysis of the target volume and relevant OAR. For the PTV, the evaluated dosimetric parameters included mean dose, $D_{1 \mathrm{cc}}, V_{95} \%$, and $V_{110 \%}$, where $D_{1 \mathrm{cc}}$ was defined as the maximum dose to $1 \mathrm{~cm}^{3}$ of PTV, and $V_{n} \%$ was defined as the percentage of PTV receiving $n \%$ of the prescribed dose. The conformity of the plans was measured with a conformity index (CI), defined as the ratio between the $95 \%$ isodose volume and the volume of the CSI PTV ( $\left.V_{95} \% / V_{\text {PTV }}\right)$. PTV heterogeneity was measured with a homogeneity index (HI), defined as the ratio between the difference of the doses delivered to 5 and $95 \%$ of the target volume and the mean dose delivered to the PTV (i.e., $\left.\left(D_{5} \%-D_{95} \%\right) / D_{\text {mean }}\right)$. For the relevant OAR, the mean dose for each organ was reported for dosimetric comparison. To further examine the quality of the RA plans, the $V_{95} \%$ in the body, excluding the PTV structure, was measured to examine the presence of high-dose regions and hot spots outside of the intended target volume. Histograms were obtained by averaging the corresponding volumes for both patients, with a bin size of $0.05 \mathrm{~Gy}$.

To evaluate the affect of a mechanical inaccuracy or a patient setup error on the quality of the RA CSI plan, an intentional error was introduced into the multi-isocenter planning technique. A displacement of $\pm 3 \mathrm{~mm}$ was introduced along the longitudinal axis of the treatment couch. This was seen as a realistic setup error, when treatment couch sag, calibration inaccuracies, and patient intrafraction motion are considered. The longitudinal error was implemented by shifting the structure set, in reference to the planned isocenters, in the treatment planning system, and the dose distributions were calculated with fixed monitor units (MU).

\section{Results}

The RA CSI plans were able to generate dose distributions with high PTV conformity and homogeneity for both patients included in the retrospective study. Examples of the dose distributions for patients $\mathrm{A}$ and $\mathrm{B}$ are illustrated in Figs. 2 and 3, respectively, and the average DVH statistics for the PTVs are listed in Table 2.

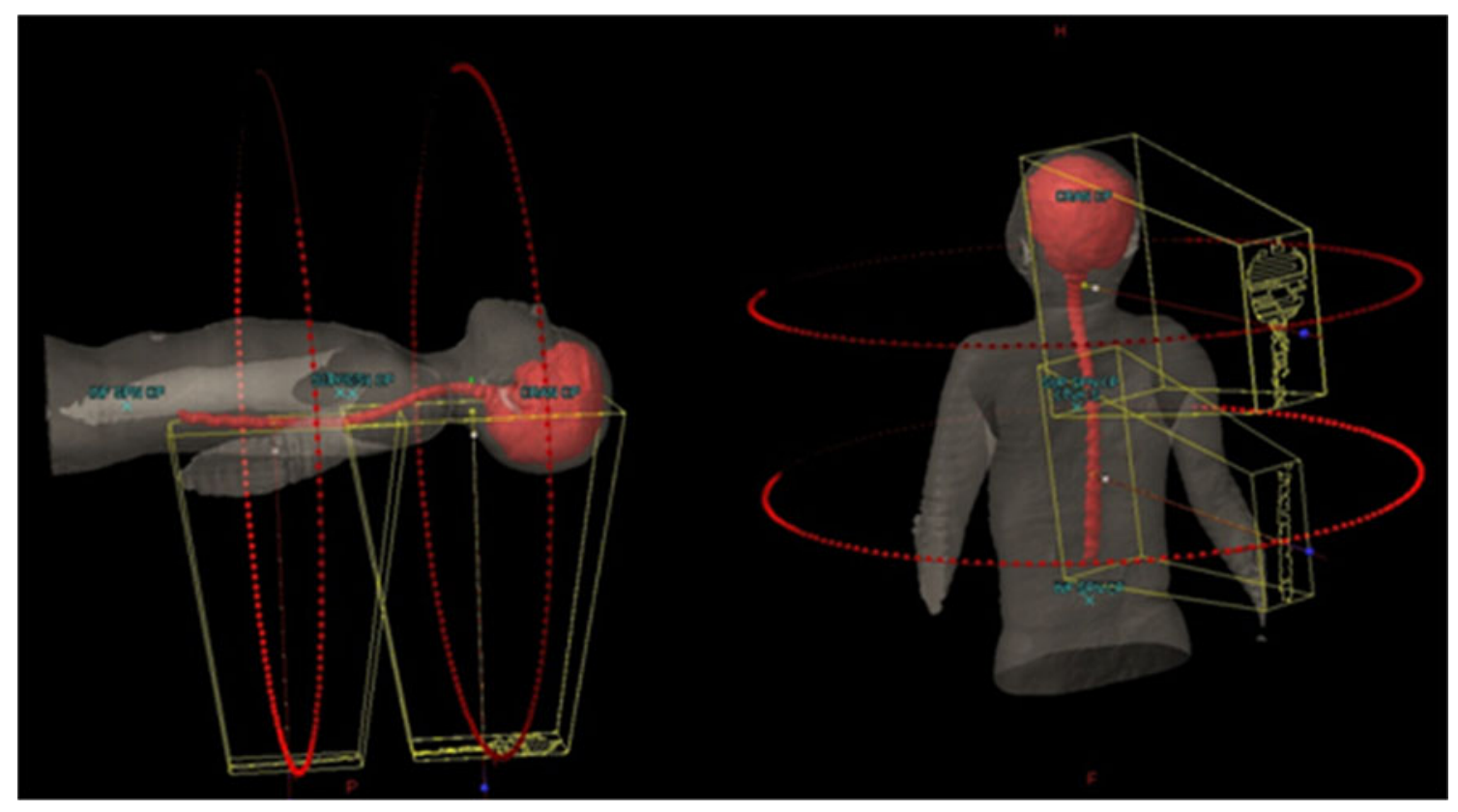

Fig. 1 RapidArc field setup with the CSI PTV illustrated (red volume) 
The CI for patients A and B were 1.00 and 0.99 , respectively, and the $\mathrm{HI}$ for the two patients were 12.7 and $11.8 \%$, respectively. The mean PTV doses for patients A and B were 102.5 and $102.1 \%$, respectively. Both patients had a $V_{110} \%$ of less than $2 \%$, and patients A and B had a $V_{107} \%$ of 12.6 and $7.8 \%$, respectively.

The RA CSI technique also produced plans with acceptable OAR dose statistics. Mean doses to the lenses, lungs, heart, spleen, kidneys, and normal tissues are displayed in Table 2.

The evaluation of mechanical inaccuracy or patient setup error indicated that minor discrepancies in the intended setup result in relatively small dosimetric changes throughout the CSI target volume. The simulation of the $\pm 3-\mathrm{mm}$ longitudinal error in the treatment plan resulted in a maximum overdose of $107.0 \%$ and maximum underdose of $85.0 \%$, both in the area of the spinal canal. The corresponding dose errors in the cranial-spinal junction areas were less than $\pm 10 \%$ for both patients. The results of the setup error analysis are graphically depicted in Fig. 4.

\section{Discussion}

In this study, we developed a novel RA technique to deliver supine CSI. The dosimetric results illustrated



Fig. 2 Dose distribution (sagittal and axial slices) for patient A. Color dose ranges from $50 \%$ to $115 \%$ of the prescription dose

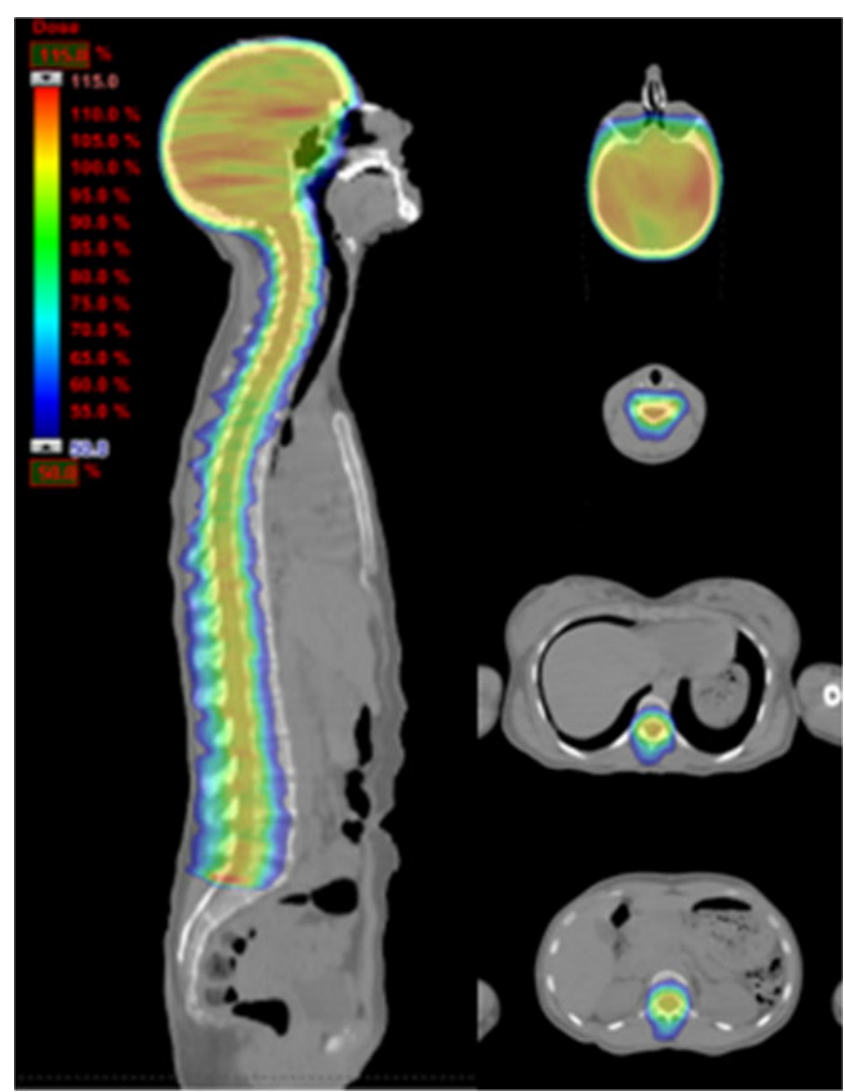

Fig. 3 Dose distribution (sagittal and axial slices) for patient B. Color dose ranges from $50 \%$ to $115 \%$ of the prescription dose

Table 2 Dose statistics for the PTV, OAR, and normal tissue

\begin{tabular}{llcc}
\hline Target & Parameter & Patient A & Patient B \\
\hline PTV & $D_{\text {mean }}(\%)$ & 102.5 & 102.1 \\
& $D_{5 \%}(\%)$ & 108 & 108 \\
& $D_{95 \%}(\%)$ & 95 & 95 \\
& $D_{1 \mathrm{cc}}(\%)$ & 117 & 114 \\
& $V_{95 \%}(\%)$ & 95 & 95 \\
& $V_{107} \%(\%)$ & 12.6 & 7.8 \\
& $V_{110 \%(\%)}(1.9$ & 0.8 \\
& HI (\%) & 12.7 & 11.8 \\
& CI & 1.00 & 0.99 \\
Normal tissue & $V_{95 \%}(\%)$ & 0.29 & 0.25 \\
& $V_{95 \%(c c)}(56$ & 95 \\
Lens & Mean (Gy) & 7.1 & 7.0 \\
Thyroid & Mean (Gy) & 7.2 & 12.3 \\
Lung & Mean (Gy) & 5.3 & 6.4 \\
Heart & Mean (Gy) & 3.6 & 4.6 \\
Liver & Mean (Gy) & 3.8 & 4.4 \\
Spleen & Mean (Gy) & 4.3 & 5.0 \\
Kidney & Mean (Gy) & 6.3 & 6.2 \\
Esophagus & Mean (Gy) & 8.1 & 11.0 \\
Left optic nerve & Mean (Gy) & 21.1 & 28.0 \\
Right optic nerve & Mean (Gy) & 21.4 & 28.0 \\
\hline
\end{tabular}



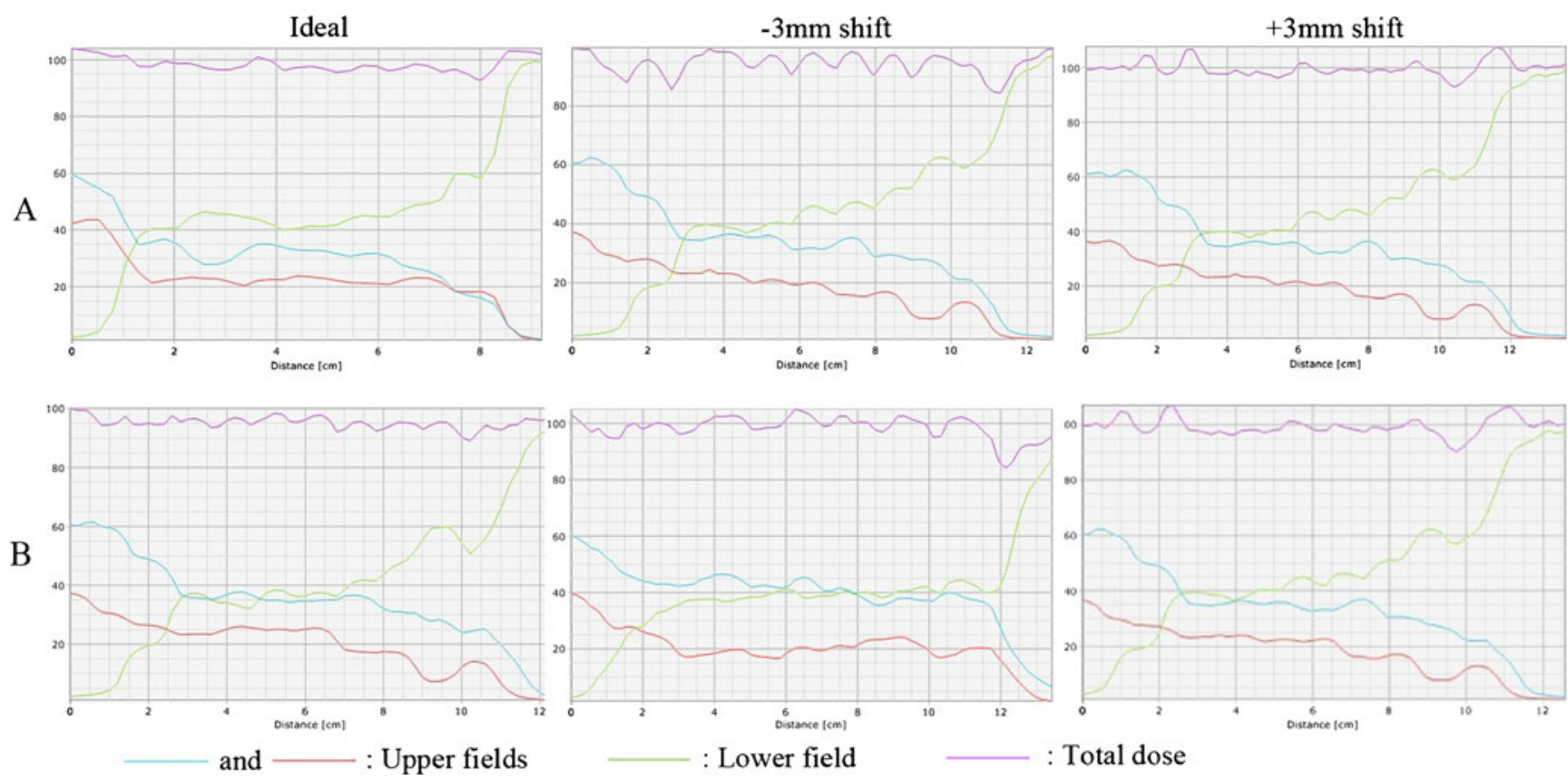

Fig. 4 The effect of deliberate longitudinal setup errors $( \pm 3 \mathrm{~mm})$ on the intended CSI dose distribution in the area of the spinal cord for patients A and $\mathrm{B}$

that a homogeneous and conformal dose to the brain and spinal canal could be achieved, while limiting the dose to the relevant OARs, with this innovative multiple-isocenter technique. By using RA, the field junction matching difficulties were alleviated with the use of overlapping fields/arcs, where the dose contribution from each arc was automatically calculated during the optimization process.

Lee et al. have developed and compared a Smartarcbased VMAT technique with conventional radiotherapy for CSI (23.4 Gy/13 fractions) [12]. They reported a median (range) conformity index of $1.22(1.09-1.45)$ and 1.04 (1.03-1.07), and a significant reduction of mean and maximum doses to the heart, thyroid, esophagus, optic nerves, and eyes, when compared with conventional plans. Their heterogeneity index, defined as the ratio of the maximum dose to $1 \mathrm{~cm}^{3}$ of PTV and the prescription dose, was slightly better than the same parameter from our results. However, the CI from our study was greatly improved, and the mean doses to OAR were lower. In general, highly conformal and homogeneous dose distributions are achievable with VMAT due to its high angular sampling rate of radiation beams [13]. The variation of parameters depends on different weightings and priorities used during planning. For our study, the goal was to create highly conformal plans, with an acceptable small compromise of dose homogeneity.

Conventional IMRT and tomotherapy have also been investigated in CSI [14-17]. Seppälä et al. have developed a method to improve target dose homogeneity for craniospinal irradiation using a single plan with dynamic split-field IMRT [14]. The results showed that improved dose homogeneity in the target volume was achieved with the split-field IMRT, when compared to that of conventional 3DCRT.

Sharma et al. [15] dosimetrically compared 3DCRT, linac-based IMRT, and tomotherapy techniques for the treatment of CSI for pediatric and adolescent patients. The conclusion of the study was that tomotherapy was technically easier and potentially dosimetrically favorable, when compared to the other techniques. The most significant problems of both tomotherapy and IMRT techniques were the increase in whole-body integral doses and prolonged delivery times. Both of these problems have further raised concerns about intrafraction motion. Our study showed that RA can achieve highly conformal dose distributions that are comparable to the helical tomotherapy techniques, with fewer MUs.

Mechanical accuracy is essential for CSI treatments, especially when the total dose is delivered in a single plan with high-dose gradients. Typical mechanical tolerances are on the order of $\pm 1 \mathrm{~mm}$ for an MLC position, couch positions, and isocenter movement during gantry rotation. Patient motion has to also be taken into account when considering the technical uncertainties. Typical misalignment errors may vary between 0 and $3 \mathrm{~mm}$. In this study, an error of $\pm 3 \mathrm{~mm}$ along the longitudinal axis of the treatment couch was introduced to the plan. As seen in Fig. 3, a shallow dose gradient was achieved in the overlapping region for each arc 
automatically. The \pm 3 -mm shift had only a small effect on the dose distribution with an error less than $\pm 10 \%$ of the calculated spinal cord dose. In practice, the field match errors are random and assumed to be smaller than $3 \mathrm{~mm}$. Therefore, the overall dose uncertainties in a RA CSI treatment are expected to be much lower than the reported $10 \%$.

There is a growing concern about secondary malignancies in CSI, especially for pediatric patients. Compared with conventional 3DCRT techniques, the doses to the thyroid, larynx, heart, and esophagus were reduced by RA. However, the doses to the large surrounding volumes, and other organs such as the lungs and kidneys, were increased. The biological effect of the increased volume receiving a lower dose is still not clear. Whether an intermediate dose to certain organs is safer than a lower dose to multiple organs is currently unknown when considering the risk of secondary malignancies. However, it has been shown that IMRT may increase the risk of secondary cancer due to the high number of MUs required to deliver a plan $[18,19]$. The dose to normal organs not in the proximity of the PTV arises largely from collimator transmission and scatter radiation from the linac, and this dose is proportional to the number of MUs. The RA VMAT technique is designed to use MUs more efficiently, decreasing the significance of this problem. The MUs used in this study are similar to that of 3DCRT and are much less than conventional IMRT. Therefore, RA might play a role in reducing overall risks of secondary cancers for patients with a long life expectancy, when compared with conventional IMRT.

Another important advantage of RA, compared with more complex delivery techniques such as IMRT, is the relative ease of implementing the therapy. The planning time is generally less than $30 \mathrm{~min}$, and the time for treatment delivery is reduced from $10-16 \mathrm{~min}$ for IMRT or tomotherapy to only a few minutes. This results in less time for potential motion errors while the patient is on the treatment couch.

In this study, the CTV of CSI was defined as the entire leptomeningeal region and the cranial contents (brain and meninges) and the spinal canal. Whether the CTV should include the spinal canal only or the entire vertebra body is an active issue in debate. It is a balance between the risk of uneven bone growth and the reduction in risk of damage to all other organs.

\section{Conclusion}

This study provides a practically useful planning method for RA treatment of CSI and illustrates the ability of RA to generate highly conformal and homogeneous treatment plans for pediatric and adult CSI, while limiting the dose to the relevant OARs. It is shown that the resultant RA dose distribution is much less sensitive to the uncertainties caused by mechanical inaccuracies or patient setup errors. RA CSI appears to be a dosimetrically advantageous, faster, and safer technique to deliver CSI treatments.

Acknowledgments We would like to thank the K.-S. Li Foundation and NIH (1R01 CA104205) for supporting the research. We also wish to acknowledge useful input from Edward Mok and Albert Koong during the course of this work.

Conflict of interest The authors declare that they have no conflict of interest.

\section{References}

1. Parker WA, Freeman CR (2006) A simple technique for craniospinal radiotherapy in the supine position. Radiother Oncol 78:217-222

2. Michalski JM, Klein EE, Gerber R (2002) Method to plan, administer, and verify supine craniospinal irradiation. J Appl Clin Med Phys 3:310-316

3. Yom SS, Frija EK, Mahajan A et al (2007) Field-in-field technique with intrafractionally modulated junction shifts for craniospinal irradiation. Int J Radiat Oncol Biol Phys 69:1193-1198

4. Otto K (2008) Volumetric modulated arc therapy: IMRT in a single arc. Med Phys 35:310-317

5. Shaffer R, Nichol AM, Vollans E et al (2010) A comparison of volumetric modulated arc therapy and conventional intensitymodulated radiotherapy for frontal and temporal high-grade gliomas. Int J Radiat Oncol Biol Phys 76(4):1177-1184

6. Clivio A, Fogliata A, Franzetti-Pellanda A et al (2009) Volumetricmodulated arc radiotherapy for carcinomas of the anal canal: a treatment planning comparison with fixed field IMRT. Radiother Oncol 92(1):118-124

7. Vanetti E, Clivio A, Nicolini G et al (2009) Volumetric modulated arc radiotherapy for carcinomas of the oro-pharynx, hypo-pharynx and larynx: a treatment planning comparison with fixed field IMRT. Radiother Oncol 92(1):111-117

8. Verbakel WF, Cuijpers JP, Hoffmans D et al (2009) Volumetric intensity-modulated arc therapy vs. conventional IMRT in headand-neck cancer: a comparative planning and dosimetric study. Int J Radiat Oncol Biol Phys 74(1):252-259

9. Johnston M, Clifford S, Bromley R et al (2011) Volumetricmodulated arc therapy in head and neck radiotherapy: a planning comparison using simultaneous integrated boost for nasopharynx and oropharynx carcinoma. Clin Oncol (R Coll Radiol) 23(8):503-511

10. Doornaert P, Verbakel WF, Bieker M et al (2011) RapidArc planning and delivery in patients with locally advanced head-and-neck cancer undergoing chemoradiotherapy. Int J Radiat Oncol Biol Phys 79(2):429-435

11. Lee CT, Bilton SD, Famiglietti RM et al (2005) Treatment planning with protons for pediatric retinoblastoma, medulloblastoma, and pelvic sarcoma: how do protons compare with other conformal techniques? Int J Radiat Oncol Biol Phys 63(2):362-372

12. Lee YK, Brooks CJ, Bedford JL et al (2012) Development and evaluation of multiple isocentric volumetric modulated arc therapy technique for craniospinal axis radiotherapy planning. Int J Radiat Oncol Biol Phys 82(1):495-496

13. Li R, Xing L (2011) Bridging the gap between IMRT and VMAT: dense angularly sampled and sparse intensity modulated radiation therapy. Med Phys 38(9):4912-4919

14. Seppälä J, Kulmala J, Lindholm P et al (2010) A method to improve target dose homogeneity of craniospinal irradiation using dynamic split field IMRT. Radiother Oncol 96(2):209-215 
15. Sharma DS, Gupta T, Jalali R et al (2009) High-precision radiotherapy for craniospinal irradiation: evaluation of threedimensional conformal radiotherapy, intensity-modulated radiation therapy and helical tomotherapy. Br J Radiol 82(984):10001009

16. Parker W, Filion E, Roberge D et al (2007) Intensity-modulated radiotherapy for craniospinal irradiation: target volume considerations, dose constraints, and competing risks. Int J Radiat Oncol Biol Phys 69(1):251-257
17. Pai Panandiker A, Ning H, Likhacheva A et al (2007) Craniospinal irradiation with spinal IMRT to improve target homogeneity. Int J Radiat Oncol Biol Phys 68(5):1402-1409

18. Followill D, Geis P, Boyer A (1997) Estimates of whole-body dose equivalent produced by beam intensity modulated conformal therapy. Int J Radiat Oncol Biol Phys 38:667-672

19. Hall EJ, Wuu CS (2003) Radiation-induced second cancers: the impact of 3D-CRT and IMRT. Int J Radiat Oncol Biol Phys 56 (1):83-88 